\title{
Research on Spherical Subsurface Imaging with Holographic Penetrating Radar
}

\author{
Mintai $\mathrm{Li}^{1, a}$, Chunlin Huang ${ }^{1, b}$ \\ ${ }^{1}$ School of Electronic Science and Engineering, National University of Defense Technology, \\ Changsha 210073, China; \\ anjutigerlee@163.com, bhclg@126.com
}

Keywords: Spherical interface, Subsurface Imaging, Holographic Penetrating Radar.

\begin{abstract}
This paper aims at subsurface imaging a target buried in a spherical homogeneous medium. In order to study it, a monochromatic holographic penetrating radar with high resolution is involved. Different from the target in the plane media, spherical ones always result in spherical interference fringes which awfully deteriorate the imaging quality. To solve this problem, this paper propose two methods and make a brief comparison. As we all know, radar echo mainly consists of direct wave and target echo. Due to the symmetry of the spherical interface, the direct wave and target echo are quite different in spatial spectrum domain distribution, and thus by using the first method, one can extract the target echo. The radar is placed right above the spherical interface, and samples thousands times along homocentric path. Therefore, direct wave varies very slowly and target echo is a sparse matrix. Then, a smoothing filtering can extract the direct wave. As a consequence, the remaining part is nearly the target echo. Furthermore, experiments are designed and validate the availability of proposed methods. What's more, a brief comparison between two methods is provided.
\end{abstract}

\section{Introduction}

In order to detect and image the targets buried in the medium, nondestructive subsurface imaging is gradually gaining its popularity. This technology has been widely used in archaeology, security, iatrical diagnosis, nondestructive evaluation, and so on. Among the multitudinous subsurface imaging radars, monochromatic holographic radar consolidates its status by several exclusive superiorities. First of all, it is immune to the dispersive media which might be the fatal disturbance for wideband radars. In dispersive media, electromagnetic waves with different frequencies radiate with different velocities. Thus, the backscattering signal is such extremely distorted that radar cannot reveal the location and shape of the target. Secondly, a monochromatic system is much easier to build up. For a wideband radar, a wideband source and a wideband antenna are indispensable. Thirdly, such a monochromatic radar radiates continuous wave so that it scans much faster than other radars. Accordingly, authors choose this radar to carry on their research and all the radars involved in this paper are monochromatic. But each coin has two sides, monochromatic subsurface radars are much more sensitive to the interface than the wideband ones.

As long as one talking about subsurface imaging, the interface (usually the air-media interface) which is a crucial factor, cannot be ignored. When subsurface imaging is done through a non-planar interface, regular or irregular interference fringes always occur [1]. Currently, both theoretical researches and practical experiments focus more on planar interfaces than on non-planar ones. But in practical applications, the media are mostly not planar. Diao Qi-long analyzed the cause of the interference when subsurface imaging through an inclined plane, and formulated the distance between fringes. In his paper, he claimed that it is interference of direct wave that leads to these fringes. On the basis of Diao's conclusion, Xu Sheng-zhi generalized this theory to cylindrical interface and proposed a novel method which is capable of eliminating the direct wave and removing the interference fringes in the final imaging result [2]. In Xu's opinion, the interface's contours are straight lines parallel to the generatrix. Once this generatrix denoted by X-axis, direct wave would be the function of the unique variable $\mathrm{y}$, and then through a 2D Furrier transformation, 
direct wave in spatial spectrum is a Dirac function about the wave number $k_{x}$. Then a filter in spatial spectrum domain is designed to remove direct wave.

On the basis of Diao's and Xu's theories, this paper generalizes subsurface imaging with holographic penetrating radar to spherical interface. In order to extract the target echo, two methods are proposed respectively and compared to each other.

\section{Imaging Model}

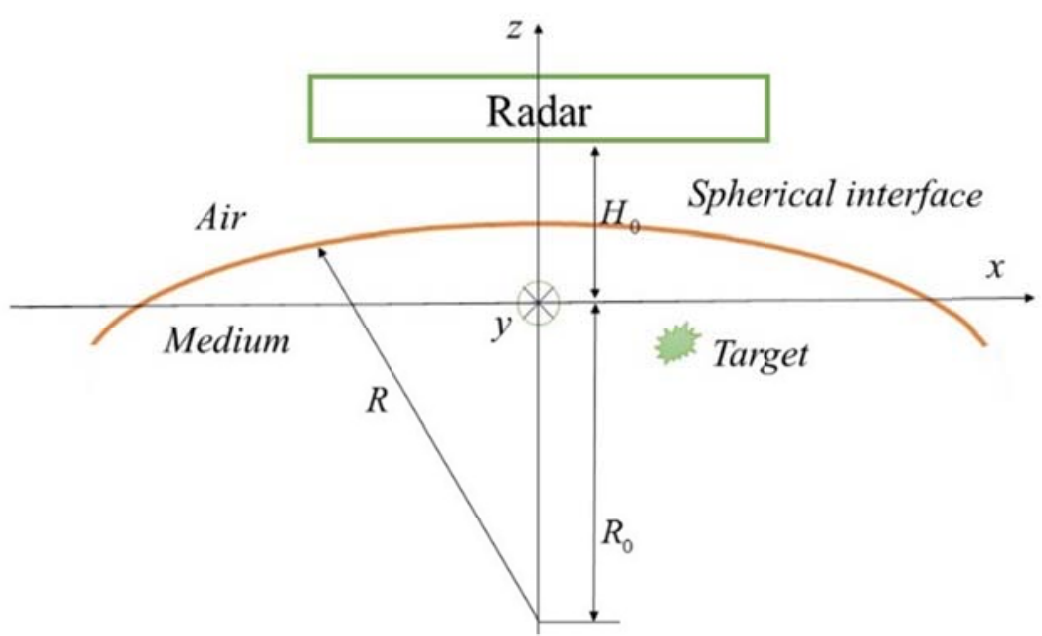

Fig. 1 Spherical subsurface imaging model

As is shown in Fig. 1, the target is assumed buried beneath a spherical air-medium interface. The medium is homogeneous. And the size of the target is much smaller compared to the radius of spherical interface and the scanned area. The radar is placed right above the interface so that the scanned area is of central symmetry. Antennae rotate along a series of homocentric circles with proportional radiuses and sample more than 100 times proportionally every turn.

Considering the geometric characteristic of the scanned area and antennae's path, polar coordinates are the most suitable and are chosen undoubtedly. Direct wave, scattered by the interface, can de denoted by $y_{s}(\rho, \theta)$, and the target echo is denoted by $y_{t}(\rho, \theta)$ respectively. The echo received by the radar can be regarded as the sum of these two parts:

$$
y(\rho, \theta)=y_{s}(\rho, \theta)+y_{t}(\rho, \theta) .
$$

Due to the interface scattering and medium attenuation, the amplitude of target echo is much smaller than that of direct wave. According to Diao's view, the direct wave can be written as

$$
y_{s}(\rho, \theta)=S(f(\rho, \theta)) e^{-j 2 \pi f \frac{\sqrt[2]{\left(H_{0}-f(\rho, \theta)\right)^{2}-\left(\frac{D}{2}\right)^{2}}}{v}-\varphi} .
$$

$\mathrm{D}$ is the distance between transmitter and receiver. In this paper,

$$
f(\rho, \theta)=\sqrt{R^{2}-\rho^{2}}-R_{0} \text {. }
$$

What is crucial is extracting the target echo from the received echo or removing the direct wave from the received echo. The following methods filter out the direct wave in different domains.

\section{Spatial spectrum domain}

Theoretical reasoning. Due to geometric symmetry of scanned area of the interface, the direct wave is constant when only angular coordinate $\theta$ varies, in another word,

$y_{s}(\rho, \theta)=y_{s}(\rho)$.

After a 2D Furrier transformation, the spatial spectrum of direct wave can be written as, 


$$
\begin{aligned}
Y_{s}\left(k_{\rho}, k_{\theta}\right) & =\iint_{\int} y_{s}(\rho, \theta) e^{-j k_{\rho} \rho-j k_{\theta} \theta} d \rho d \theta=\iint y_{s}(\rho) e^{-j k_{\rho} \rho-j k_{\theta} \theta} d \rho d \theta \\
& =\int y_{s}(\rho) e^{-j k_{\rho} \rho} d \rho \int e^{-j k_{\theta} \theta} d \theta .
\end{aligned}
$$

Apparently, $Y_{s}$ yields 0 as long as $k_{\theta} \neq 0$, because

$$
\int e^{-j k_{\theta} \theta} d \theta=\left\{\begin{array}{ll}
0 & k_{\theta} \neq 0 \\
\infty & k_{\theta}=0
\end{array}\right. \text {. }
$$

Practically, since the domain of integration is finite, previous integral yields a large finite number instead of infinite when the wave number is zero. Since neither the target's shape nor its location coordinates is of symmetry, target echo in spatial spectrum domain distributes over a wide area. Due to distribution difference in spatial spectrum domain, a filter can be designed to remove the direct wave, which is

$$
H\left(k_{\rho}, k_{\theta}\right)=\left\{\begin{array}{ll}
1 & k_{\theta} \neq 0 \\
0 & k_{\theta}=0
\end{array} .\right.
$$

Multiplying the echo's spectrum by this filter could undoubtedly eliminate direct wave, but also lose some target information where the wave number is zero. Therefore, a regression is carried out to compensate this information loss. When all procedures above done, the remaining echo data is mostly the target echo and could reveal the target clearly with the help of imaging algorithm.

Experiment. Now that the radar and target are placed as Fig.1 shows, let radar execute a polar coordinate proportional sampling. The Radar radiate a monochromatic continuous wave to detect the target covered by an ABS board with spherical upper surface. The object used as target is a plastic which seems the capital letter $T$ in shape. As Fig.3 (a) shows, both branches of the target plastic are about $3.8 \mathrm{~cm}$ long and $0.6 \mathrm{~cm}$ wide. What's more, two holes with diameter of $0.2 \mathrm{~cm}$ can be found on each branch. Fig.3 (b) illustrate and make a contrast of the spectrum distribution of the echo before and after filtering. The upper figure is the echo original spectrum; as Equation (5) implies, echo spectrum yields a line because target echo is much weaker than direct wave. The lower figure reveals that, once the direct wave is filtered out in spectrum domain, the remaining part is weaker and mostly the target echo. Fig.3 (c) presents the imaging result without filtering, that is, it is generated by the echo data of the upper figure in Fig.3 (b). Expected circular interference fringes can be found and deteriorate the image. Once the direct wave is filtered out, the imaging result would be what Fig.3 (d) shows and the circular interference fringes would be eliminated completely.

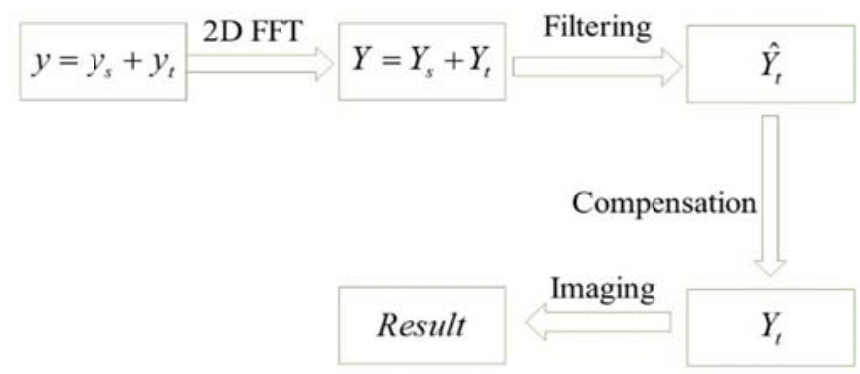

Fig. 2 Filtering procedures in spatial spectrum domain 


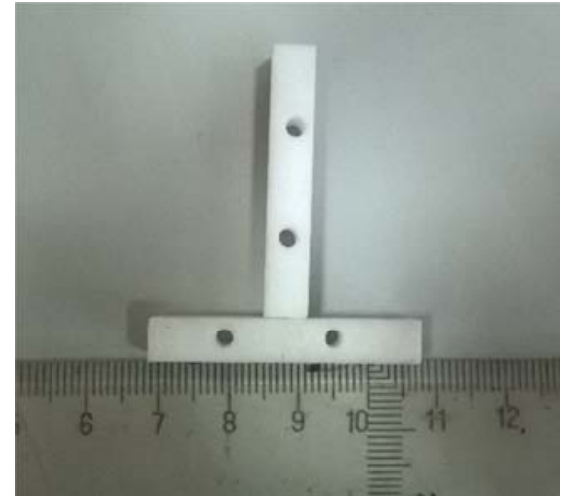

(a) Target shape and size

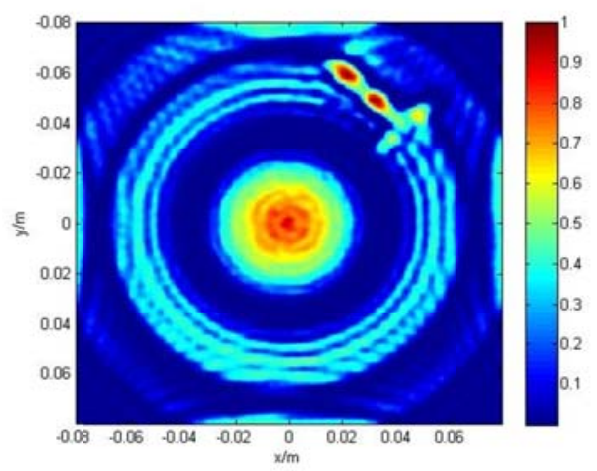

(c) Imaging result without filtering
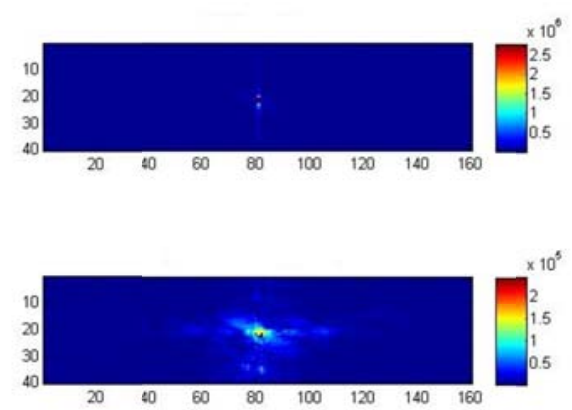

(b) Spectrum before and after filtering

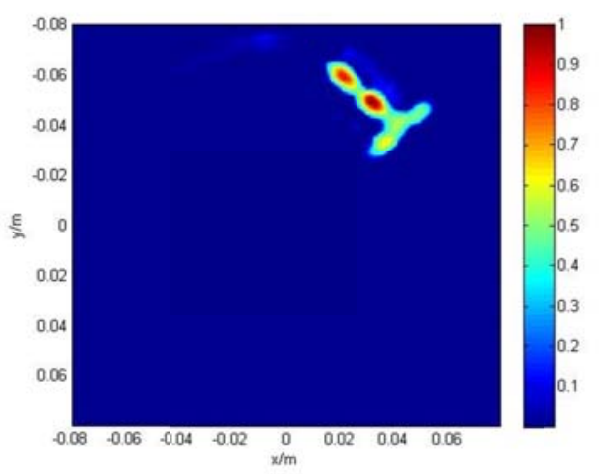

(d) Imaging result after filtering

Fig. 3 Subsurface imaging with spectrum filtering

\section{Spatial domain}

Theoretical reasoning. The radar and target are still placed as Fig.1 shows. The antennae move along series of homocentric circles so that radar is capable of sampling at every fixed angle in every turn. Radar store the echo data at every sampling place in order. Therefore, received echo data can be written as a matrix

$$
R_{M \times N}=R_{s}+R_{t} \text {, }
$$

where $M$ represents the antennae rotate $M$ turns, and $N$ means the sampling times in each turn. A row in $R$ means echo data from a common circular sampling. For example, the first row in the data matrix means the series of echo data in first turn with a radius $\rho_{1}$. According to Equation (4), direct wave is constant in first row. The similar conclusion can be drawn in arbitrary row. Therefore, for an arbitrary positive integer $x$ no more than $N$,

$$
R_{s}\left(x, y_{1}\right)=R_{s}\left(x, y_{2}\right)
$$

Where $y_{1}$ and $y_{2}$ are arbitrary positive integers no more than $M$. 


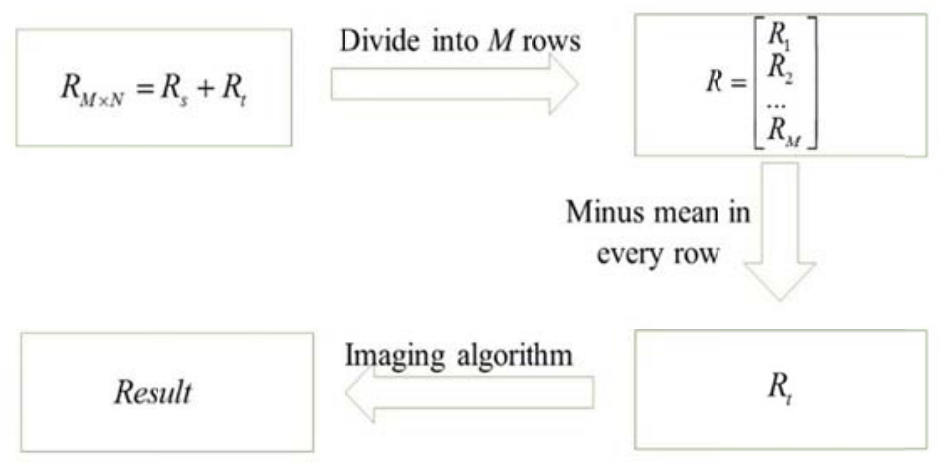

Fig. 4 Filtering procedures in spatial domain

Meanwhile, the target echo is a sparse matrix because target is small compared to the scanned area. What's more, due to the attenuation in the media, target echo is usually much weaker than direct wave. As we know, once a matrix minus the mean of all its elements, its constant component would be removed. Retrospect Equation (8) and divide echo data matrix $R$ into $M$ rows. According to previous reasoning, if every row minus the mean of its elements, the direct wave would be removed. According to Equation (8), the remaining part of echo is mostly the target echo. Then without the disturbance of direct wave, radar could output a perfect image result.

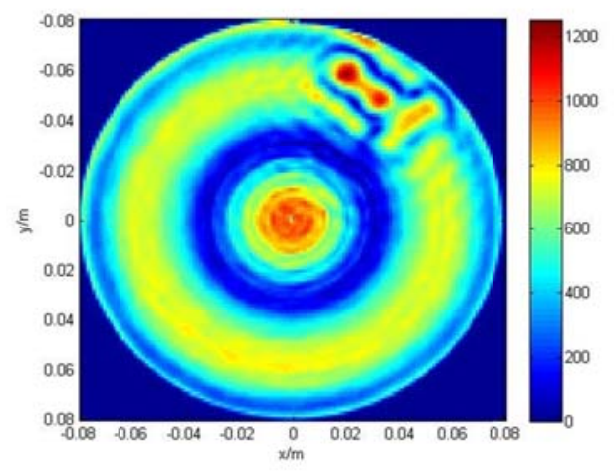

(a) Original echo

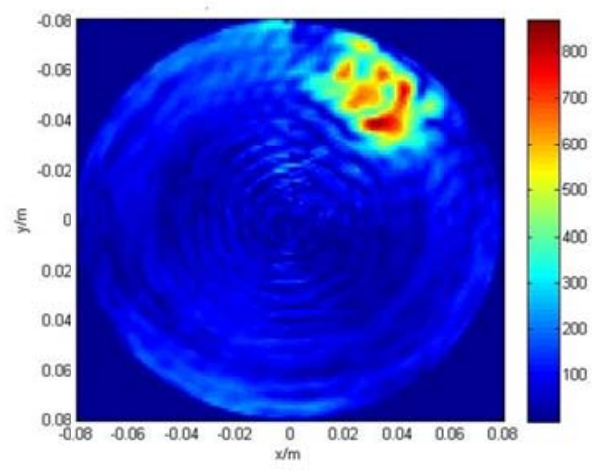

(b) Filtered echo

Fig.5 Filtering in spatial domain

Experiment. The experiment setup is the same as that in former section. The same target and ABS board, but different filtering procedures. By filtering the direct wave out in spatial domain, a similar imaging result to former section comes out naturally. Fig.5 exhibits the contrast of original echo and filtered echo. Both of them are interpolated and presented in rectangular coordinates.

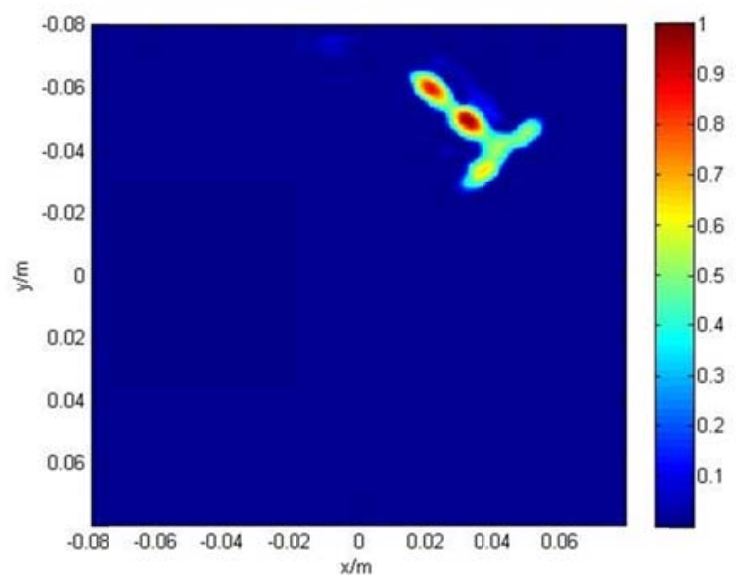

Fig. 6 Imaging result of spatial filtered echo 
As is shown in Fig.5 (a), direct wave is almost constant in each one circular sampling path, which demonstrated the implication of Equation (9). In another words, the each row of the matrix shown by Equation (8) is nearly constant except the disturbance of sparse target echo. Once these rows minus their own expectations, the direct wave would be removed while only would target echo remain as Fig. 5(b) shows. Applying imaging algorithm to the remaining target echo, one may acquire the final imaging result without interference fringes (see Fig.6). Compared to the final result in former section (see Fig.3 (d)), the performance of filtering in spatial domain is parallel to that of filtering in spatial spectrum domain. What's more, as fierce scattering sources, small holes in plastic are highlighted in the final results.

\section{Summary}

Based on the former researches, this paper proposes two filtering methods to remove the direct wave when spherical subsurface imaging. These methods process the echo data in spatial spectrum domain and in spatial domain respectively. Experiments have already demonstrated their availability. Furthermore, proposed methods are also suitable for other curve surface media of central symmetry, because the fundamental precondition is as Equation (4) implies. Since the echo data are processed in spectrum domain, a 2D Furrier transformation is compulsory. Considering the imaging algorithm is based on spectrum domain, a 2D Furrier transformation is compulsory for second method when direct wave removed. Therefore, two methods are ordinarily equivalent in time cost.

By proposing two method to filter out direct wave, this paper generalizes subsurface imaging with monochromatic holographic penetrating radar to spherical interface. But exclusive available interface shape and restrict radar placement prohibit these method from wide usage. Therefore, an urgent improvement must be achieved for multiple interfaces.

\section{References}

[1] Diao Qi-Long, Huang Chun-Lin, Restraining parasitic interface fringe phenomenon in detection imaging through the medium with inclined angle, Acta Phys. Sin. Vol.61, No.21 (2012).

[2] Xu Sheng-zhi, Huang Chun-lin, Liu Tao, Qin Yao, Lu Min, A study of removing interference fringes on cylindrical subsurface imaging with continuous wave penetrating radar, Journal of Electronics and Information Technology,Vol.37,No.11, Nov 2015.

[3] C Huang, T Liu, The impact of an uneven medium surface in holographic penetrating imaging and a method to eliminate the interference, IEEE Region 10 Conference- 2013.

[4] Sang-Eun Park, Sophie Allain, Surface Roughness and Microwave Surface Scattering of High-Resolution Imaging Radar, IEEE GEOSCIENCE AND SENSING LETTERS, VOL.12,NO.4,APRIL 2015

[5] D.Sukhanov, K.Zavyalova. Three-dimensional non-contact subsurface radiotomography through a non-planar interface between media,15th International Conference on Ground Penetrating Radar-GPR2014

[6] L Capineri, F Fiesoli, C Windsor, "Holographic radar: A strategy for uneven surfaces", International Conference on Ground Penetrating Radar - GPR2012.

[7] Paul D.Walker,Mark R.Bell. "Noniterative Techniques for GPR Imaging Through a Nonplanar Air-Ground Interface", IEEE TRANSACTIONS ON GEOSCIENCE AND REMOTE SENSING,VOL.40,NO.10,OCTOBER 2002. 